\title{
Publisher Correction: The computational cost of active information sampling before decision-making under uncertainty
}

Pierre Petitet (D), Bahaaeddin Attaallah (D), Sanjay G. Manohar (D) and Masud Husain (D)

Correction to: Nature Human Behaviour https://doi.org/10.1038/s41562-021-01116-6, published online 27 May 2021.

In the version of this article initially published, errors occurred in Figs. 1a,c and 4b. In Fig. 1a,c, labels for equation elements were omitted, and a close-parenthesis was missing in Fig. 1c. In Fig. 4a, the equation contained an incorrect symbol ( $\int$ should have been $\left.f\right)$; and in Fig. 4b, colored shading was misplaced. The errors have been corrected in the PDF and HTML versions of this article. 


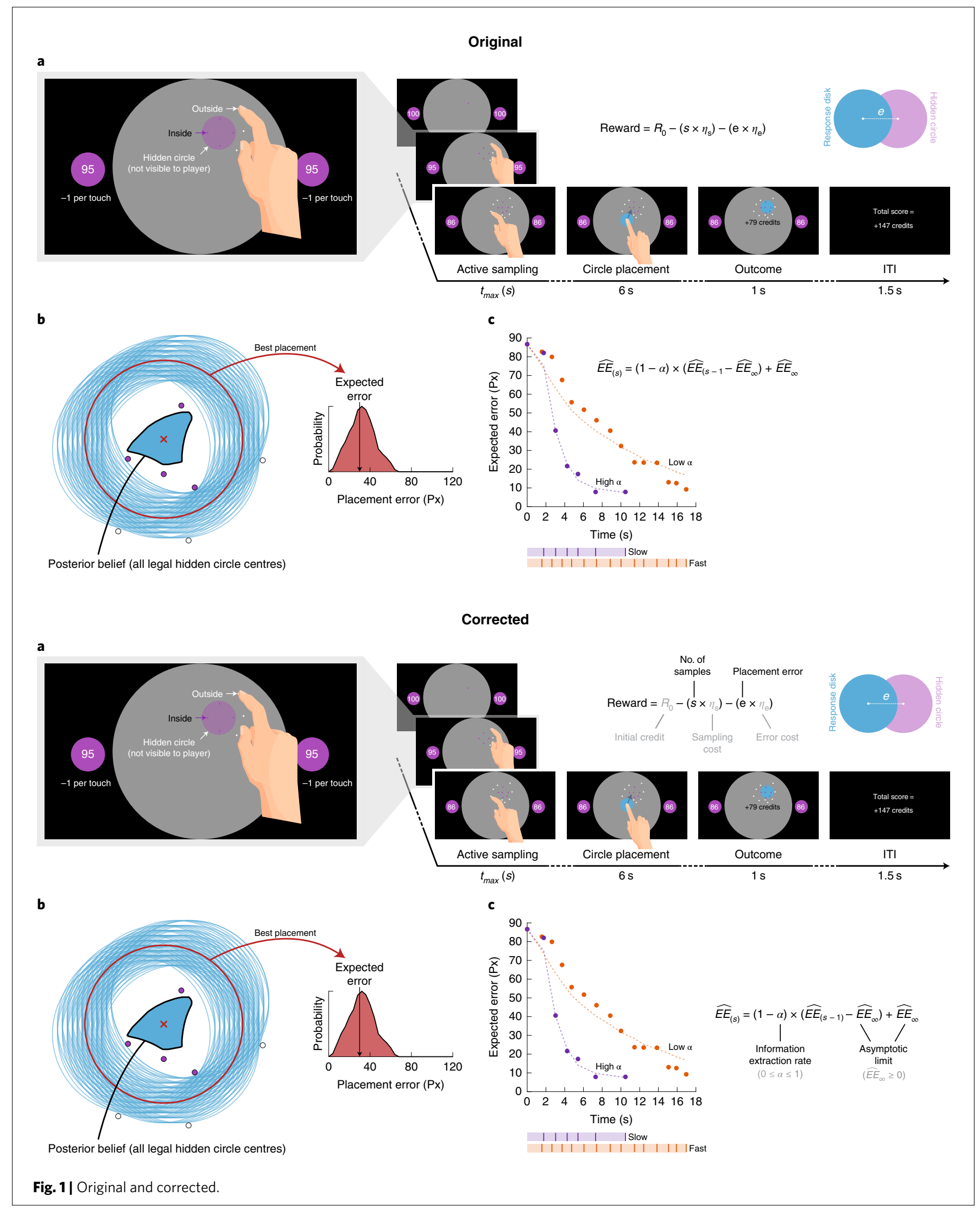




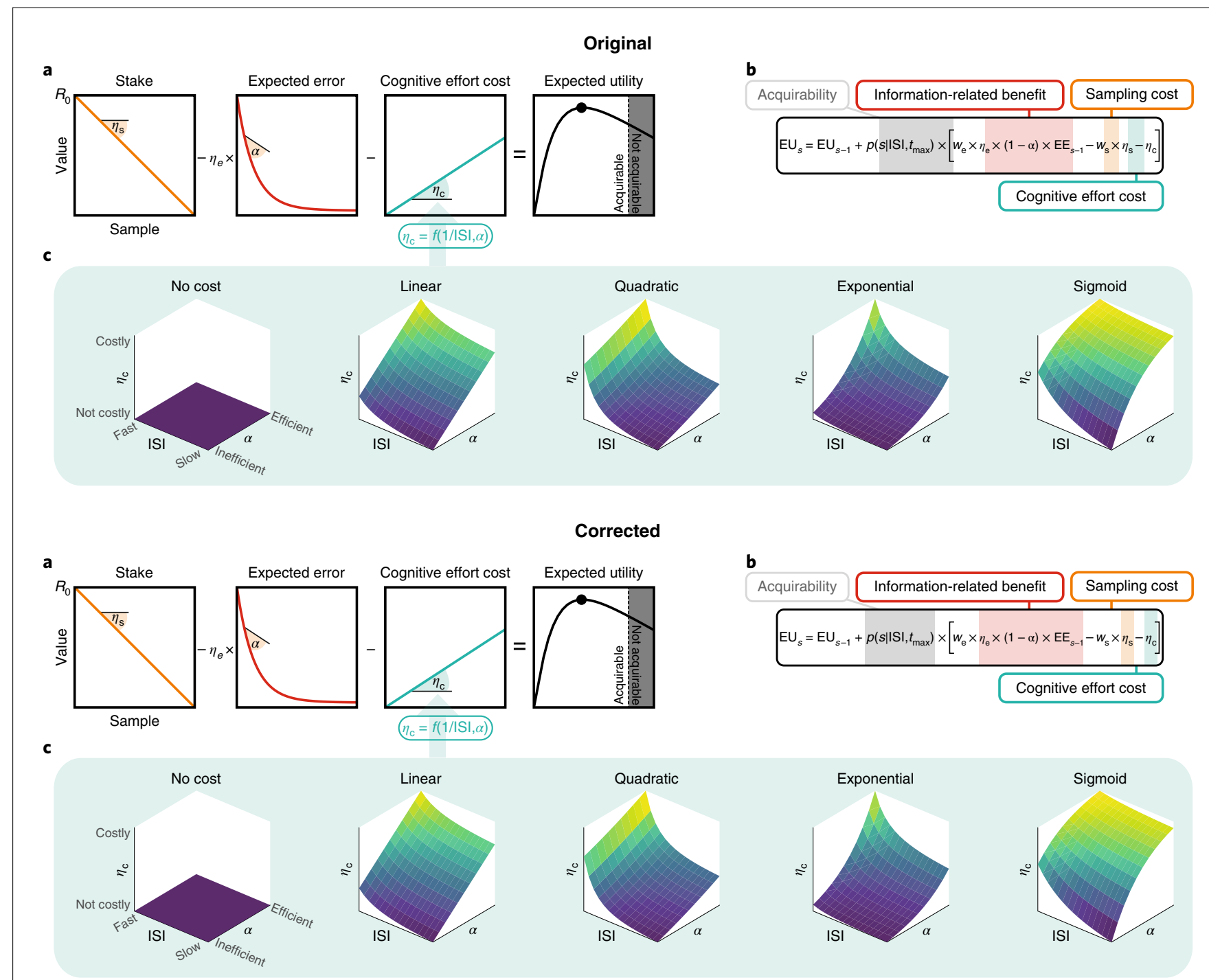

Fig. 4 | Original and corrected.

Published online: 13 July 2021

https://doi.org/10.1038/s41562-021-01165-x

๑ The Author(s), under exclusive licence to Springer Nature Limited 2021 